\title{
Rat spatial memory: Both working and reference memory are resistant to retroactive interference
}

\author{
KHANH TRAN \\ Moorhead Senior High School, Moorhead, Minnesota \\ and \\ WILLIAM W. BEATTY \\ North Dakota State University, Fargo, North Dakota
}

\begin{abstract}
Previous studies of the spatial working memory (WM) of rats trained in radial mazes indicate that WM is highly resistant to retroactive interference (RI). In the present experiment, a procedure that permitted simultaneous assessment of WM and reference memory (RM) was used to train rats in an eight-arm radial maze. Interpolation of testing on a second radial maze during a brief delay increased both WM and RM error rates, albeit modestly. But when the rats were subsequently trained on two interpolated mazes during the retention interval, no increase in WM or RM error rates was seen. In the radial maze, both RM and WM are relatively resistant to RI.
\end{abstract}

Recent experiments using the radial maze have revealed that the rat's spatial working memory (WM) system has high capacity (Olton, Collison, \& Werz, 1977; Roberts, 1979) as well as high accuracy that persists for as long as $8 \mathrm{~h}$ (Beatty \& Shavalia, 1980b; Knowlton, McGowan, Olton, \& Gamzu, in press).

Spatial WM in rats is also highly resistant to retroactive interference (RI). Maki, Brokofsky, and Berg (1979) exposed rats to various events during a 2-min-long retention interval imposed between the fourth and fifth choices in an eight-arm radial maze. The interpolated events included exposure to lights, sounds, or odors, feeding in the maze, and testing on a four-arm maze located in another room. None of these events produced RI. Using a similar approach, Beatty and Shavalia (1980a) interpolated training on a second eight-arm maze at varying times during a 4-h-long retention interval. They also observed no RI. When required to remember spatial information from two eight-arm mazes concurrently, their rats performed as accurately as when they had to remember only the places they had visited in one maze. Roberts (1981) varied the number of eight-arm-maze experiences that were interpolated between the first four and subsequent choices on an eight-arm maze. Significant RI was observed only if training on three interpolated mazes occurred during the retention interval for the first maze. If only one maze was interpolated, Roberts observed essentially no RI at all. This result obtained even when the test

K.T. participated in the Minority High School Student Research Apprenticeship Program at North Dakota State University; the program is supported by NIH Grant S03 RR 03344 from the Division of Research Resources. Address reprint requests to the second author at: Department of Psychology, North Dakota State University, Fargo, ND 58105. maze and the interpolated maze were located in the same room, a procedure that should have maximized interference by maximizing the similarity of stimuli controlling responses on the two mazes.

Traditional theories of memory maintain that WM is appreciably more susceptible to interference than is reference memory (RM). Nearly "foolproof" demonstrations of RI for events held in WM can be found in virtually every contemporary text in introductory psychology, but few if any illustrations of interference effects involving RM are to be found in such sources.

From the foregoing, it follows that: Given the remarkable resistance to RI of the rat's spatial WM, its spatial RM should be virtually impervious to RI. In the present experiment, we tested this prediction using a variation of the procedure developed by Olton and Papas (1979), which permits simultaneous assessment of WM and RM in the radial maze.

\section{Animals}

\section{METHOD}

The subjects were 11 male albino rats of a Sprague-Dawley strain purchased from the Holtzman Company, Madison, Wisconsin at 2.5 months of age. They were caged singly and given free access to water in an air-conditioned animal room that was illuminated from 0800 $2100 \mathrm{~h}$ by overhead fluorescent fixtures. The rats were fed Purina Lab Chow in an amount sufficient to maintain body weight at $85 \%$ of the free-feeding level, adjusted for growth. All testing occurred during the daylight portion of the light-dark cycle.

\footnotetext{
Apparatus

Three radial-arm mazes were employed; they were located in different rooms. The test rooms differed in size and location within the laboratory. All rooms were outfitted with a rich variety of extramaze cues, but these cues differed from room to room. The mazes arbitrarily designated Maze 1 and Maze 2 were the same 8 -arm mazes used by Beatty and Shavalia (1980a). Maze 3 was a 12-arm maze, described elsewhere
} 
(Beatty, Bierley, \& Boyd, 1984). All three mazes were made of wood painted white and were equipped with black plastic sidewalls that extended the length of the arms. The guillotine doors located at the junction of the arms and the cental hub were made of black plastic on Mazes 1 and 2 and of clear plasic on Maze 3. At the end of the arms in each maze were identical receptacles for reinforcers. On Maze 1 the receptacles were simply recesses in the floor of the arms; on Maze 2 the receptacles were aluminum cups; on Maze 3 the receptacles were black plastic cups.

\section{Procedure}

Prior to the start of training on any maze, a set of arms was designated to be baited for each rat (four arms in Mazes 1 and 2, six arms in Maze 3). The set of baited arms varied from rat to rat but remained constant for any single animal. Assignment of the set of baited arms was at random but with the following restrictions: (1) The baited set could not consist of four (or six) arms that were all adjacent to one another; and (2) the pattern of baited arms for a particular rat could not be the same for Mazes 1 and 2. The latter constraint was imposed to preclude the possibility that the two mazes could be solved by adopting a common response pattern. All baited arms in the three mazes contained a single $190-\mathrm{mg}$ Noyes pellet at the start of the daily session. Baits were not replenished during the session.

Pretraining on all mazes was conducted in the following way. The doors to the baited arms were raised while the doors to the unbaited arms remained lowered. The rat was given 10 min to locate and consume all of the baits; pretraining on a particular maze continued until all 11 rats had consumed all baits within $10 \mathrm{~min}$. Pretraining on Maze 1 began on Day 1 of the experiment. Pretraining on Maze 2 began on Day 16. Pretraining on Maze 3 began on Day 43. Pretraining required 9 days on Maze 1, 3 days on Maze 2, and 1 day on Maze 3.

Formal training on Maze 1 began on the day after all rats had met the pretraining criterion. The rats were forced to make their first two choices (or three choices on Maze 3) to arms selected at random from their set of baited arms. The particular arms they were forced to choose varied from day to day. After the rats had completed their forced choices, all arms were opened immediately. The session continued until the rat found the remaining food pellets. Entries into unbaited arms were considered RM errors; reentries into baited arms were considered WM errors.

During initial training on each maze, no delay was imposed between the forced choices and the subsequent free choices. By Day 27 the rats had achieved accurate performance on Maze 1; RM error rates were consistently less than one per session, and WM errors almost never occurred. Accordingly, for the next five sessions a 2-min delay was imposed between the two forced choices and subsequent free choices in Maze 1. During the delay, the rats were confined to a small chamber in an adjacent room. Training with no delay continued on Maze 2. Beginning on Day 33 and continuing for 10 days, the effects of interpolating training on Maze 2 on retention of Maze 1 were studied. Alternate days were designated as interference or control sessions. On interference days, the rat received: (1) two forced choices in Maze 1; (2) two forced choices in Maze 2; (3) free-choice retention in Maze 1; and (4) free-choice retention in Maze 2 in that sequence. On control sessions, that rat received: (1) two forced choices in Maze 1; (2) 2-min detainment in the small chamber; (3) free-choice retention in Maze 1; and (4) standard training with 0 delay in Maze 2 . The delay in Step 2 was set at 2 min because this interval was slightly longer than the average time required to transport the rats from Maze 1 to Maze 2, administer two forced choices in Maze 2, and return the animals to Maze 1.

Starting on Day 50 and continuing for the next 7 days, the length of the delay between the two forced and subsequent free choices in Maze 1 was extended to $5 \mathrm{~min}$. Testing on Mazes 2 and 3 continued with no delay imposed between the initial forced choices and subsequent free choices. Beginning on Day 57 and continuing for the next 10 days, interference and control sessions were given on alternate days. During interference sessions the rats received: (1) two forced choices in Maze 1; (2) two forced choices in Maze 2; (3) three forced choices in Maze 3; (4) free-choice retention test in Maze 1; (5) free-choice retention test in Maze 2; and (6) free-choice retention test in Maze 3. On control ses- sions, the rats received: (1) two forced choices in Maze 1; (2) 5-min delay in the chamber as described above; (3) free-choice retention in Maze 1; (4) standard testing with no delay in Maze 2; and (5) standard testing with no delay in Maze 3. The 5-min delay imposed on control sessions was slightly longer than the average time required to complete the forced choices in Mazes 2 and 3 and transport the animals from one maze to another.

\section{RESULTS AND DISCUSSION}

Interpolation of forced-choice training on a single eightarm maze produced a slight but significant increase in the number of WM and RM errors. During the interference test sessions, WM errors averaged 0.13 per session versus 0.05 per session during control (i.e., 2-min-delay) sessions $[t(10)=2.39, \mathrm{p}<.05]$. A similar pattern was observed for RM errors: The rats averaged 0.62 errors per session during interference tests versus 0.33 errors per session on control sessions $[\mathrm{t}(10)=2.31, \mathrm{p}<.05]$. On control (i.e., 0-delay) sessions for Maze 2, the rats averaged $0.04 \mathrm{WM}$ and $0.36 \mathrm{RM}$ errors per session, suggesting that they had acquired and could utilize the basic rules governing reward delivery in the second maze.

The small but significant interference effect observed when training on Maze 2 was interpolated between the first two and subsequent choices on Maze 1 was quite unexpected. If $\mathrm{RI}$ is a consistent phenomenon under these conditions, then increasing the number of interpolated maze tasks should increase the magnitude of the interference effects. This was not the case.

When the rats were trained on two other mazes during the 5-min retention interval for Maze 1, WM errors per session averaged 0.04 during interference tests versus 0.02 for control (i.e., 5-min-delay) tests, whereas RM errors per session averaged 0.24 and 0.13 for interference and control tests, respectively (ts < 1.07). On control (i.e., 0 -delay) days, the mean number of WM errors per session was 0.05 on Maze 2 and 0.24 on Maze 3, whereas the mean number of RM errors per session was 0.07 on Maze 2 and 1.60 on Maze 3. The higher number of errors of both types on Maze 3 probably reflects the greater difficulty of the 12-arm maze as well as the fact that the rats had fewer training sessions on this task. Relative to their average performance over the first five sessions on Maze 3 (means $=0.82 \mathrm{WM}$ and $4.22 \mathrm{RM}$ errors per session), their performance on control tests was reliably better $[\operatorname{ts}(10)>2.65$, ps $<.01]$. Hence, it is reasonable to conclude that the rats maintained their mastery of the general rules governing solution of Mazes 2 and 3 during the interference tests.

Since performance on control sessions during the second series of interference tests improved from the already excellent performance on the first series, sensitivity to RI should have been very high on both measures. The failure to observe RI under these conditions suggests that RI is negligible for both RM and WM, at least in the radial maze. The weak RI effects observed during the first series of interference tests reflect either chance differences 
or extremely modest effects that can be observed only when the rats are not thoroughly trained on the test maze.

Regardless of which interpretation of the latter issue one favors, the present findings emphasize again that rat spatial memory is nearly impervious to RI. Consequently, interpretations of the disruptive effects of drugs on spatial memory that emphasize pharmacological potentiation of interference (e.g., Burešova \& Bures, 1982) are called into question.

\section{REFERENCES}

Beatty, W. W., Bierley, R. A., \& Boyd, J. (1984). Amphetamine disrupts both working and reference memories of rats trained in a radial maze. Behavioral and Neural Biology, 42, 169-176.

Beatty, W. W., \& Shavalia, D. A. (1980a). Rat spatial memory: Resistance to retroactive interference at long retention intervals. Animal Learning \& Behavior, 8, 550-552.

Beatty, W. W., \& Shavalia, D. A. (1980b). Spatial working memory in rats: Time course of working memory and effect of anesthetics. Behavioral and Neural Biology, 28, 454-462.

BUREŠVAA, O., \& BUREš, J. (1982). Radial maze as a tool for assessing the effects of drugs on the working memory of rats. Psychopharmacology, 77, 268-271.

Knowlton, B., McGowan, M., Olton, D. S., \& Gamzu, E. (in press). Hippocampal stimulation disrupts spatial working memory eight hours after acquisition. Behavioral and Neural Biology.

MAKI, W. S., BROKOFSKY, S., \& BERG, B. (1979). Spatial memory in rats: Resistance to retroactive interference. Animal Learning \& Behavior, 7, 25-30.

Olton, D. S., Collison, C., \& Werz, M. A. (1977). Spatial memory and radial arm performance of rats. Learning and Motivation, 8 , 298-314.

Olton, D. S., \& PaPas, B. C. (1979). Spatial memory and hippocampal function. Neuropsychologia, 17, 669-682.

Roberts, W. A. (1979). Spatial memory in the rat on a hierarchical maze. Learning and Motivation, 10, 117-140.

ROBERTS, W. A. (1981). Retroactive inhibition in rat spatial memory. Animal Learning \& Behavior, 9, 566-574.

(Manuscript received for publication October 8, 1984.) 